\title{
TRANSMISIÓN DE PRECIOS INTERNACIONALES EN EL MERCADO LACTEO CHILENO
}

\author{
INTERNATIONAL PRICE TRASMISSION IN THE CHILEAN DAIRY \\ MARKET
}

\author{
Ariel Arcos Catalán ${ }^{1 *}$ \\ ${ }^{1}$ Universidad Católica del Norte, Angamos 0610, Antofagasta, Chile. \\ * Autor para correspondencia E-mail: ariel.arcos@alumnos.ucn.cl
}

\begin{abstract}
RESUMEN
En una economía abierta, como la de Chile, los precios internacionales influyen sobre los precios domésticos. Esta relación puede dar indicios de distorsión en el mercado cuando la transmisión de precios no es instantánea. En este caso, resulta relevante estudiar esta relación en el mercado lácteo chileno con la finalidad de adoptar políticas de corrección de las fallas de mercado. El objetivo de esta investigación fue determinar la medida en que se relacionan los precios de exportación e importación de lácteos con el precio pagado a productores lecheros en Chile, entre los años 1990-2018. La metodología utilizada fue el análisis de cointegración y Modelo de Corrección de Errores (ECM). Mediante la aplicación del test de cointegración se obtiene que el precio nacional presenta una relación de equilibrio de largo plazo con el precio de exportación, no así con el precio de importación. Los resultados de la estimación del ECM entre precio nacional y precio de exportación indican que tanto los coeficientes de ajuste de corto plazo como de largo plazo son estadísticamente significativos, hecho que evidencia que la referencia para la fijación de precios por parte de la industria en Chile es el precio de exportación. La magnitud de estos parámetros revela que las variaciones del precio de exportación no se transmiten instantáneamente hacia el precio nacional.
\end{abstract}

Palabras clave: leche, transmisión de precios, integración de mercados, cointegración, ley de un solo precio.

\section{ABSTRACT}

In an open economy like Chile, international prices influence domestic prices. This relationship can result in market distortions when price transmission is not instantaneous. In this sense, it becomes relevant to study this relationship in the Chilean dairy market to adopt policies to correct market failures. The objective of this research was to determine the extent to which export and import prices of dairy products are related to the price paid to dairy producers in Chile between the years 1990-2018. The methodology used was cointegration analysis and Error Correction Model (ECM). By applying the cointegration test, it was observed that that there is a long-term equilibrium relationship between local and export prices, but not with import price. The results of the ECM estimation between local and export prices indicate that both short-term and long-term adjustment coefficients are statistically significant. This indicates that the reference for industry pricing in Chile is export price. The magnitude of these parameters reveals that the variations in export price are not transmitted instantaneously to local price.

Key words: milk, price transmission, market integration, cointegration, law of one price. 


\section{INTRODUCCION}

En Chile, el mercado lácteo es altamente concentrado y tiene características de oligopsonio, con muchos productores lecheros que actúan como oferentes y pocas empresas lecheras como demandantes (Díaz et al., 2007; Labra et al., 2017). Además, este mercado tiene la característica particular que el commodity transado tiene el carácter de perecedero, lo que dificulta el poder de negociación de los oferentes (Díaz et al., 2007). De esta forma, las empresas demandantes de leche tienen un mayor poder de negociación sobre los precios pagados a productor. Cabe entonces preguntarse: ¿en base a qué criterios fija la industria los precios pagados a productores? Algunas de las respuestas son la calidad de la leche y la estacionalidad de la producción a lo largo del año. Pero, además, tal como lo establece la teoría económica, en una economía abierta los precios internacionales son un referente para la fijación de precios internos de economías pequeñas, que no influyen en el precio mundial, pero que si actúan como tomadores de precio como es el caso de Chile (Engler y Nahuelhual, 2008).

En un mercado abierto de commodities que es perfectamente competitivo, los cambios exógenos de precios internacionales debiesen trasmitirse instantáneamente al precio nacional. Situación que reflejaría que no hay poder de arbitraje y donde las únicas diferencias en el precio entre países deberían ocasionarse por los costos de transporte según la Ley de Un Solo Precio (Serra y Goodwin, 2003). Surge entonces la necesidad de estudiar el mercado de commodities, en particular el mercado lácteo en Chile, por las características mencionadas. De esta forma, se puede revelar el grado de distorsión existente que permita orientar de mejor forma algunas políticas públicas tendientes a regular dichas fallas de mercado.

En estudios previos que analizan la transmisión de precios de commodities, tanto en Chile como en otros países, son ampliamente utilizados los precios nacionales, así como los precios internacionales, entendidos estos últimos como los precios de importación y/o exportación (Engler y Nahuelhual, 2008; Newton, 2016; Labra et al., 2017). Con estas variables se estiman las relaciones de cointegración entre las series de precios, es decir, si siguen una relación de largo plazo común. Cumpliéndose esta condición es posible estimar el grado de integración entre los mercados, así como la velocidad en que se transmiten los cambios en los precios internacionales (shoks exógenos) a los precios nacionales mediante la estimación de Modelos de
Corrección de Error (ECM) (Jaramillo y Benítez, 2016). Así es posible conocer el grado del poder de mercado que tienen las empresas demandantes a la hora de fijar los precios pagados a los oferentes.

En este sentido, la investigación de Engler y Nahuelhual (2003) indica que en Chile, el precio de importación de lácteos es tomado como referencia por las empresas lácteas nacionales para establecer el precio pagado a productor en el periodo 1996-2001. Estos mismos resultados se dan también para el periodo 1990-2002 (Nahuelhual y Engler, 2004).

También para el mercado lácteo chileno, Díaz et al. (2007) concluyeron que en el periodo comprendido entre 1979 y 2005 son los precios de importación de lácteos la referencia para fijar los precios pagados a productor en Chile. No obstante, estimando un modelo econométrico con cambio estructural, indica que entre 1989 y 2005 el precio de referencia para la industria láctea chilena podría haber sido el precio de exportación, dado el paulatino incremento de las exportaciones de lácteos desde 1990, junto a una reducción gradual de las importaciones chilenas de lácteos.

Posteriormente, Engler y Nahuelhual (2008) estimaron que en Chile el precio de las importaciones de lácteos es tomado como referencia para la fijación del precio interno en el periodo 1990-2000; mientras que desde 2001 a 2007 el precio de exportación pasó a ser el nuevo referente para la industria láctea nacional. Sin embargo, la significancia estadística de la relación entre precio de exportación y precio doméstico, no les permitió concluir de manera robusta ni la dirección ni la magnitud del efecto del precio de exportación hacia el precio nacional entre 2001 y 2007. Estos resultados justifican que en la presente investigación se evalúe tanto la relación de importación como exportaciones hacia el precio pagado a productor en Chile, en un periodo que incluye una mayor extensión de años posteriores a 2001.

Los estudios antes mencionados, indicaron también que los precios nacionales e internacionales se encuentran cointegrados, es decir, siguen una relación de largo plazo común. Dándose tal situación, resulta importante señalar que los Modelos de Corrección de Error (ECM) son utilizados para analizar la integración de mercados, así como también la transmisión dinámica de precios.

De acuerdo a la Fig. 1, desde 2004 en adelante, Chile pasa a ser un exportador neto a excepción de los últimos cuatro años. Si a dicho comportamiento se suman los indicios de un posible cambio de precio de referencia indicado por Díaz et al. (2007) y los hallazgos de Engler 


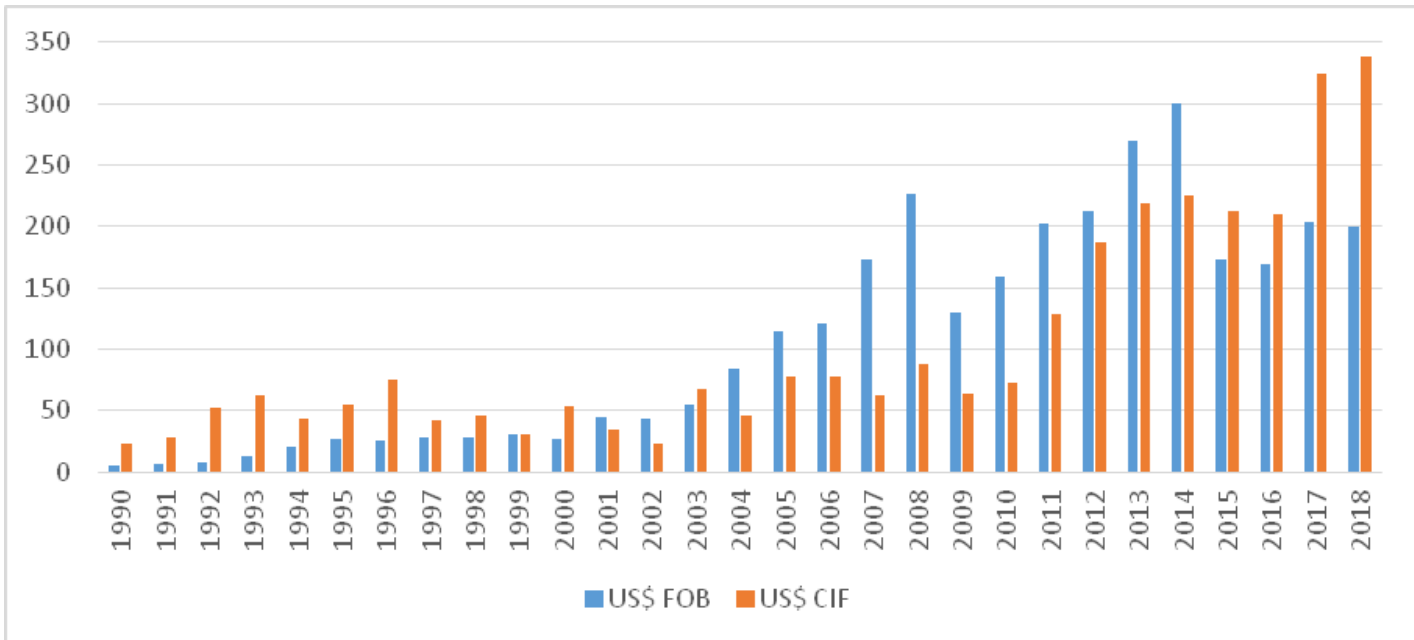

Fig. 1. Exportación e importación de lácteos en Chile (Millones de dólares)

Fig. 1. Dairy product exports and imports in Chile (Million dollars)

Fuente: Elaboración propia en base a ODEPA (2017b)

y Nahuelhual (2008), se podría esperar que desde dichos resultados hasta 2018, el precio de exportación manifieste un efecto significativo que permita concluir que es el precio de referencia para la industria nacional, más que el precio de importación.

Como se aprecia en la Fig. 2, los precios internacionales y precios nacionales muestran variaciones similares, hecho que da indicios de una posible relación de largo plazo entre el precio doméstico y los precios internacionales, lo que se investiga a lo largo de la presente investigación.

En base a los antecedentes mencionados, el objetivo de la presente investigación fue determinar en qué medida los precios de importación y exportación se relacionan con los precios pagados a productor por las plantas lecheras chilenas en el periodo 1990-2018. Al mismo tiempo se busca verificar si efectivamente hay un cambio de precio de referencia hacia los precios de exportación.

\section{MATERIALES Y METODOS}

\section{Fuentes de información}

La información utilizada corresponde a una serie de tiempo con periodicidad anual desde 1990 al año 2018. Siendo las variables de interés el precio nacional pagado a productor por litro de leche, y el precio de las importaciones y exportaciones de lácteos.

El precio promedio pagado a productor nacional, sin Impuesto al Valor Agregado (IVA) y expresado en pesos por litro de leche en términos nominales, fue obtenido de las estadísticas de series de precios, disponibles en ODEPA (2017a). Estos precios son posteriormente expresados en pesos chilenos del año 2008. Para ello, se utilizaron las estadísticas de IPC del INE (2019a, 2019b, 2019c).

Desde las estadísticas de comercio exterior silvoagropecuario de ODEPA (2017b) fueron obtenidos tanto el precio de importación CIF (Cost Insurance and Freight) como el precio de exportación FOB (Free on Board) de lácteos expresados en dólares. Se consideran solo los tres productos más transados en el periodo según el monto de USD CIF y USD FOB en términos nominales. Estos productos corresponden a leche entera en polvo, leche condensada y queso, en el caso de exportaciones, y leche entera en polvo, leche descremada en polvo y queso, para importaciones.

Para expresar las cantidades en una medida comparable con la unidad de medida nacional, se procedió a convertir a litros de leche equivalentes todos los productos importados y exportados, de acuerdo al coeficiente de conversión disponible en el Boletín de la Leche de ODEPA (2017c).

Se utilizó el valor del dólar observado promedio anual, que se obtuvo del Banco Central de Chile (2017), para expresar los montos importados y exportados a pesos chilenos, para estimar un precio promedio ponderado de acuerdo a las cantidades transadas, quedando los precios de importación y exportación expresados en pesos por litro de leche equivalente en moneda de 2008.

De esta forma, tanto los precios nacionales como los de importación y exportación, quedan expresados en una misma unidad de medida. 


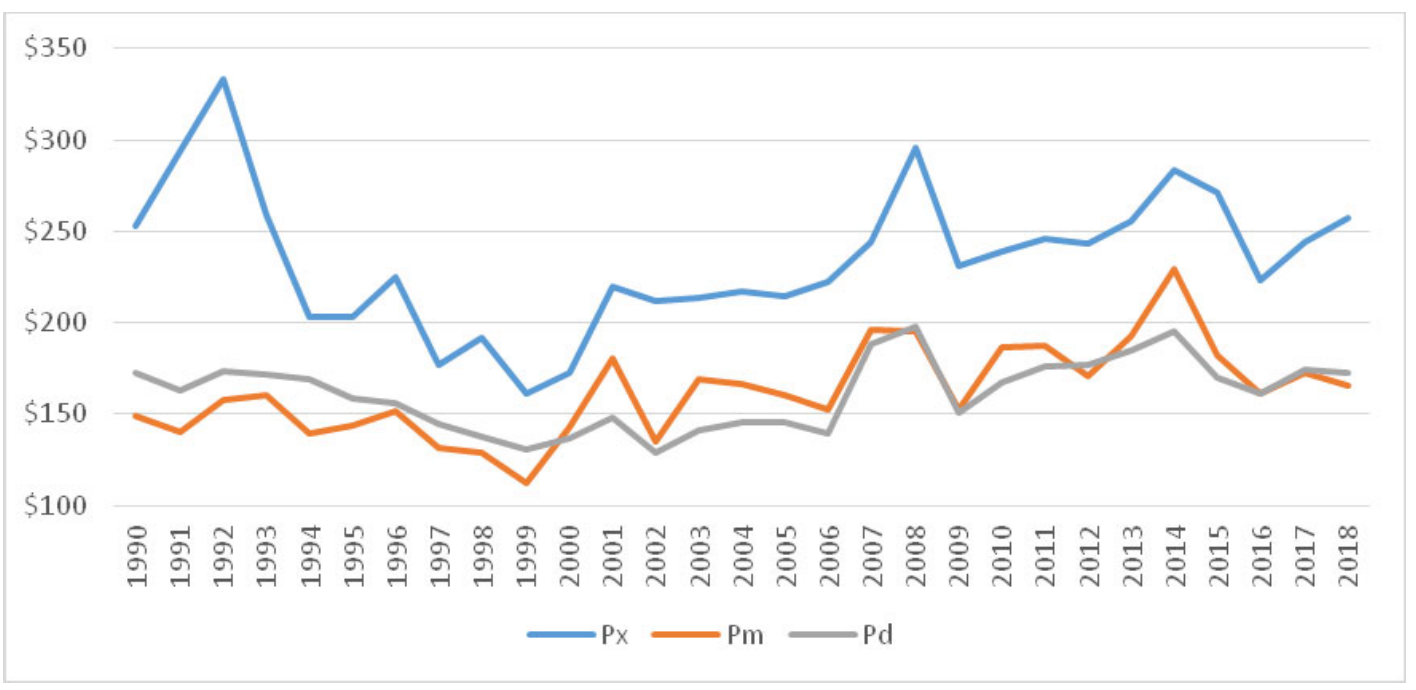

Fig. 2. Evolución del precio de importación (Pm), precio de exportación (Px) y precio nacional (Pd) por litro de leche (Pesos del año 2008)

Fig. 2. Evolution of import (Pm), export (Px) and local (Pd) prices per liter of milk (2008 Chilean pesos). Fuente: Elaboración propia en base a ODEPA (2017b).

\section{Especificación del modelo}

Para la presente investigación, se toma como referencia el trabajo de Díaz et al. (2007) quienes, a través de un Modelo de Corrección de Error (ECM), evaluaron la dinámica de transmisión de precios de las importaciones de leche hacia el precio recibido por productores lecheros de Chile, representado por la ecuación (1).

$$
\begin{aligned}
p_{t}^{d}-p_{t-1}^{d}= & \mu+\alpha\left(p_{t-1}^{w}-p_{t-1}^{d}\right)+ \\
& \beta\left(p_{t}^{w}-p_{t-1}^{w}\right)+\varepsilon_{t} \ldots(1)
\end{aligned}
$$

donde, a través del coeficiente $\alpha$, evalúan la velocidad de ajuste hacia el equilibrio de largo plazo entre el precio internacional por litro de leche $\left(p_{t}^{w}\right)$ y el precio por litro de leche pagado a productor nacional $\left(p_{t}^{d}\right)$. Mientras que a través del coeficiente $\beta$ miden el ajuste de corto plazo entre las mencionadas variables.

Cabe mencionar que los análisis de cointegración junto a los ECM son utilizados en el análisis de trasmisión de precios, donde se destacan los trabajos de Dutoit et al. (2010), quienes evaluaron la relación de precios del maíz y arroz en América Latina; Valdés et al. (2011) investigaron la integración de precios nacional e internacional en el mercado chileno del trigo; Stephens et al. (2012) evaluaron la cointegración y transmisión de precios para el mercado de tomates en Zimbabwe. Por otra parte, Baquedano y Liefert (2014) evaluaron la integración y transmisión de precios entre distintos commodities entre más de 60 países en desarrollo, y Gaetano (2015) analizó la transmisión de precios de tomate y coliflor entre distintas regiones de la Unión Europea.

En el estudio del mercado lácteo, destacan los recientes trabajos de Newton (2016), quien analizó la transmisión de precios entre Oceanía, Estados Unidos y la Unión Europea, y Popovic et al (2016), que estudiaron el mercado lácteo de Serbia.

En la presente investigación se utiliza el ECM siguiendo el procedimiento de dos etapas de Engle-Granger, el cual es representado por la ecuación (2). A través de la cual se determina la relación entre precio doméstico y precio de importación, además de la relación entre precio doméstico y precio de exportación de lácteos.

$$
\begin{gathered}
P d_{t}-P d_{t-1}=\beta_{0}+\beta_{1}\left(P i_{t}-P i_{t-1}\right)+ \\
\beta_{2} \mu_{t-1}+\varepsilon_{t} \ldots \text { (2) } \\
P d_{t}=\alpha_{0}+\alpha_{1} P i_{t}+\mu_{t}
\end{gathered}
$$

En la ecuación (2) y (3), $P d_{t}$ representa el precio pagado a productor nacional por litro de leche expresado en logaritmo natural. Por su parte $P i_{t}$ corresponde al precio de importación o exportación por litro de leche equivalente expresado en logaritmo natural.

Mientras que $\mu_{t-1}$ corresponde al término de error de la ecuación cointegrante (3) rezagado en un periodo y representa el mecanismo de corrección de error, cuyo valor es igual a 0 cuando se converge al equilibrio entre el precio nacional y el precio de importación o exportación. Mientras que cuando se da una situación de desequilibrio, es distinto de 0 .

El parámetro $\beta_{1}$ representa el efecto de 
corto plazo, es decir, cuánto del cambio en el precio de las importaciones o exportaciones, será transmitido en el periodo actual al precio nacional. Por su parte, el parámetro $\beta_{2}$ representa el término de error de equilibrio y el su valor absoluto se interpreta como la velocidad de equilibrio de largo plazo (Gujarati, 2004).

De acuerdo a Engle y Granger (1987), una de las condiciones necesarias para la aplicación de un ECM, es que las variables que componen la serie de tiempo, tengan igual grado de integración y estén cointegradas, por lo que se puede decir que las variables siguen una tendencia de largo plazo común. Para corroborar esta condición, se aplica el test de raíz unitaria Augmented Dickey Fuller (ADF) y posteriormente el contraste de cointegración de Engle-Granger.

Para la obtención de los resultados se es utilizó el software econométrico Gretl versión 1.7.1 (Cottrell y Lucchetti, 2007). Solamente se empleó el software RStudio (RStudio Team, 2016) para testear posibles cambios estructurales en modelo ECM mediante el test de Hansen y Seo (2002).

\section{RESULTADOS Y DISCUSION}

Una serie de tiempo es no estacionaria si su media y/o varianza no es constante a través del tiempo, es decir, no es integrada de orden 0 $(\mathrm{I}(0))$ por lo que al realizar una regresión entre variables de este tipo dará lugar a estimaciones sesgadas a menos que estén cointegradas y sigan una tendencia común en el largo plazo (Gujarati, 2004; Wooldridge, 2006). Estas últimas dos condiciones se cumple si: 1) dos o más variables son no estacionarias con igual grado de integración de orden 1 (I(1)), es decir, cuando la media y varianza de la primera diferencia es estacionaria, y 2) existe entre ellas una relación lineal que resulte en un residuo estacionario $\mathrm{I}(0)$ (Gujarati, 2004).

Con el objetivo de evaluar la estacionariedad en el logaritmo de las variables en estudio, se realizó la prueba de Dickey Fuller Aumentada
(ADF). En este test, se contrasta la hipótesis nula de existencia de raíz unitaria, que en caso de ser rechazada, indicaría que las variables son estacionarias. Los resultados de la aplicación del test ADF a las series temporales de precios se muestran en la Tabla 1. Estos resultados muestran que en las series de precios, el estadístico calculado tau no es estadísticamente significativo, no rechazándose la hipótesis nula de raíz unitaria, por lo que las variables presentan un comportamiento no estacionario.

Dado los resultados del test ADF aplicado las series de precios, se procedió a realizar el test $\mathrm{ADF}$ a la primera diferencia logarítmica de los precios de leche. Estos resultados se presentan en la tercera columna de la Tabla 1 e indican que las variables son estacionarias en primera diferencia, es decir, tanto los precios nacionales como internacionales son integrados de orden $1(\mathrm{I}(1))$.

Si dos variables no están cointegradas, la regresión de una sobre la otra dará como resultado una relación espuria y no habrá ninguna relación de largo plazo entre ambas variables. Por otro lado, si están cointegradas, se pueden establecer modelos dinámicos como los ECM (Wooldridge, 2006).

Para determinar si el precio nacional de leche y el precio FOB y CIF, están cointegrados, se utilizó el contraste de cointegración de Engle-Granger al logaritmo de dichas variables. El mencionado test se basa en la estimación de una regresión cointegrante entre la variable dependiente y la independiente, que en el caso de la presente investigación, corresponde a la especificación (3).

Posteriormente se realiza el contraste ADF a los residuos $\mu_{t}$ obtenidos de las regresiones estimadas en (3). El estadístico calculado para este contraste es $-2,14$ ( $p$ value 0,24 ) en el caso de la ecuación cointegrante entre precio nacional y precio CIF, aceptándose por lo tanto la existencia de raíz unitaria. Esto indica que el precio nacional y el precio de importación no muestran una relación de cointegración.

Por otro lado, el estadístico calculado para $\mu_{t}$

Tabla 1. Prueba ADF aplicada a las series precios y su primera diferencia temporal Table 1. ADF test applied to price series and its first temporary difference

\begin{tabular}{ccc}
\hline Precios & $\begin{array}{c}\text { Estadístico tau a la serie } \\
\text { de precios }\end{array}$ & $\begin{array}{c}\text { Estadístico tau primera } \\
\text { diferencia temporal de precios }\end{array}$ \\
\hline $\mathrm{Pm}$ & $-2,29$ & $-7,08^{* * * *}$ \\
$\mathrm{Px}$ & $-2,41$ & $-5,15^{* * *}$ \\
$\mathrm{Pd}$ & $-1,96$ & $-5,99^{* * *}$ \\
\hline
\end{tabular}

Nota: Test ADF considerando constante y un rezago.

*significativo al $10 \%$; ${ }^{* *}$ significativo al $5 \%$; ${ }^{* * *}$ significativo al $1 \%$

Fuente: Elaboración propia. 
obtenido de la relación cointegrante entre precio nacional y precio FOB es $-3,48$ ( $p$ value 0,01 ). Lo que implica que el precio doméstico y el precio de exportación se encuentran cointegrados.

Dados los resultados del test de cointegración se concluye que el precio de importación y precio nacional no siguen una relación de largo plazo. Por el contrario, para precio nacional y precio de exportación se cumple el teorema de representación de Granger (Engle y Granger, 1987). Es decir, estos dos precios siguen una relación de largo plazo, por lo que es posible expresar dicha relación mediante un ECM. Resultados que parecen sostener la sospecha de que el nuevo precio de referencia que tendrían las plantas lecheras para fijar el precio nacional sería el precio de exportación (Díaz et al., 2007).

Los resultados de la regresión cointegrante entre precio doméstico y precio de exportación se muestran en la Tabla 2. En dicha especificación, el coeficiente $\alpha_{1}$ representa la relación de equilibrio estático de largo plazo (Gujarati, 2004). Indicando que el precio pagado a productor nacional tendrá un incremento de $0,55 \%$ como consecuencia del aumento en un $1 \%$ en el precio de exportación de lácteos.

Los coeficientes estimados mediante la ecuación (2), se presentan en la Tabla 3, e indican que tanto el efecto de corto plazo como el ajuste de largo plazo son estadísticamente significativos. Estableciéndose entonces, un equilibrio dinámico de largo plazo entre precio nacional y precio de exportación.

En algunas ocasiones la relación de cointegración suele no estar representada por una relación lineal que estima el ECM. Al no cumplirse esta condición, se tendría una transmisión asimétrica de precios (Labra et al, 2017), que sería capturada de mejor forma con un Treshold Vector Error Correction Model (TVECM) (Serra

Tabla 2. Resultados regresión cointegrante entre precio nacional (Pd) y precio de exportación de lácteos (Px).

Table 2. Results of cointegrate regression between local (Pd) and export prices of dairy products (Px).

\begin{tabular}{lrc}
\hline & Coeficiente & Error estándar \\
\hline$\alpha_{0}$ & $2,06^{* * *}$ & 0,49 \\
$\alpha_{1}$ & $0,55^{* * *}$ & 0,09 \\
\hline \multicolumn{2}{l}{$\mathrm{N}^{\circ}$ observaciones: $29(1990-2018)$} \\
Estadístico Durbin-Watson $=1,27$ \\
$\mathrm{R}^{2}$ ajustado: 0,58
\end{tabular}

Nota: *significativo al $10 \%$; ${ }^{* *}$ significativo al $5 \%$; ${ }^{* * *}$ significativo al $1 \%$

Fuente: Elaboración propia.

Tabla 3. Resultados de estimación Modelo de Corrección de Errores entre precio nacional (Pd) y precio de exportación de lácteos (Px).

Table 3. Results of Error Correction Model estimation between local (Pd) and export prices of dairy products (Px).

\begin{tabular}{lcc}
\hline & Coeficiente & Error estándar \\
\hline$\beta_{0}$ & $-0,001$ & 0,01 \\
$\beta_{1}$ & $0,450^{* * *}$ & 0,11 \\
$\beta_{2}$ & $-0,609^{* * *}$ & 0,19 \\
\hline $\mathrm{N}^{\circ}$ observaciones: $28(1991-2018)$ & \\
$\mathrm{F}(2,25)=12,12(\mathrm{p}$ value $=0,00)$ & \\
$\mathrm{R}^{2}$ ajustado: 0,45 & \\
Estadístico Durbin-Watson $=1,84$ & \\
Contraste RESET: $\mathrm{F}(2,25)=1,69(\mathrm{p}$ value $=0,21)$ \\
Normalidad de los residuos: $\chi^{2}(2)=2,64(\mathrm{p}$ value $=0,27)$ \\
Contraste CUSUM: Estadístico Harvey-Collier $\mathrm{t}(24)=1,43(\mathrm{p}$ value $=0,17)$
\end{tabular}

Nota: *significativo al $10 \%$; ** significativo al $5 \%$; *** significativo al $1 \%$

Fuente: Elaboración propia. 
y Goodwin, 2003). Esto, porque los ajustes hacia el equilibrio de largo plazo ocurren solo en los periodos en que la relación de largo plazo se desvía del equilibrio a partir de un determinado threshold (Dempster y Tang, 2016).

Bajo la posibilidad de que la relación de cointegración no sea constante a través del periodo, se utiliza la metodología propuesta por Hansen y Seo (2002), quienes estiman un TVECM y proponen el test Supremum Lagrange Multiplier (supLM) cuya distribución asintótica se obtiene con el método bootstrap. Las hipótesis son las siguientes:

\section{$\mathrm{H}_{0}$ : Cointegración lineal}

$\mathrm{H}_{1}$ : Cointegración con threshold

La estimación del test supLM se realizó considerando un bootstrap de 2.999 simulaciones. Los resultados fueron un supLM de 12,20 con un p-value de 0,25. Estos resultados permiten rechazar la hipótesis nula, lo que significa que no existen quiebres estructurales en la relación de cointegración de la serie de precios y, por lo tanto, dicha relación queda correctamente representada por el ECM.

A juicio de Engler y Nahuelhual (2008), no existía una conclusión robusta sobre la relación y magnitud del efecto del precio de exportación hacia el precio nacional desde 2001 a 2007, dada la significancia estadística de su impacto. Sin embargo, en la presente investigación, al incorporar un periodo más extenso, después de 2007 se obtienen coeficientes estimados $\beta_{1}$ y $\beta_{2}$ significativos estadísticamente, resultados que complementan los de Engler y Nahuelhual (2008) y que les da mayor robustez.

De acuerdo al valor del parámetro $\beta_{1}$, se tiene que la variación de un $1 \%$ en el precio de exportación, genera en el mismo periodo, un incremento en el precio recibido por productores nacionales en un $0,45 \%$. Mientras que el coeficiente $\beta_{2}$ indica que ante discrepancias entre el precio nacional e internacional en un año, dicha divergencia se corrige en un $0,61 \%$ al año siguiente.

Si se comparan los coeficientes de ajuste de corto y largo plazo (en valor absoluto), se tiene que la transmisión de los precios de exportación hacia el precio nacional se caracteriza por ser trasmitido en mayor medida en el largo plazo que en el mismo periodo.

Al comparar los resultados del modelo de corrección de errores, se obtuvo un coeficiente de ajuste de corto plazo un tanto superior al hallado por Díaz et al. (2007) para el periodo 1979-2005, cuyo valor fue de 0,3. Mientras que el ajuste de largo plazo fue aún mayor si se contrasta con el 0,36 estimado por dichos autores. Hecho que indica que en el periodo 1990-2018, la trasmisión de precios de exportación en el mercado lácteo en Chile es un tanto más rápido que la transmisión de precios de importación en el periodo 19792005, no obstante, aún sigue siendo más bien un ajuste parcial que instantáneo.

Se destaca entonces que para el mercado de la leche en Chile no se cumple la ley de un solo precio, ya que no es traspasada la totalidad de las variaciones del precio de exportación hacia el precio nacional, tanto en el largo plazo como en el corto plazo. Esto podría estar explicado por el carácter oligopsonico de las plantas lecheras nacionales. Estos resultados podrían orientar la formulación de políticas públicas tendientes a corregir las distorsiones en el mercado lácteo chileno.

\section{CONCLUSIONES}

A través del análisis de cointegración se obtuvo que el precio por litro de leche pagado a productores chilenos no presenta una relación de largo plazo con el precio de importación de lácteos. Por el contrario, si presenta una relación de largo plazo con el precio de exportación que es representada en un ECM.

Tanto el coeficiente de ajuste de corto plazo como de largo plazo obtenido en el ECM resultaron ser estadísticamente significativos. Dicho resultado indica que en Chile, la industria láctea considera el precio de exportación como referencia para la fijación del precio pagado a productores chilenos.

La magnitud de los parámetros de ajuste del ECM revelan que las variaciones en el precio de exportación de lácteos no son transmitidas instantáneamente al precio pagado a los productores chilenos, sino que son transmitidas en mayor medida en el largo plazo.

\section{LITERATURA CITADA}

Banco Central de Chile. 2017. Bases de datos estadísticos. Tipos de cambio. Dólar observado. Disponible en https://si3. bcentral.cl/Siete/secure/cuadros/arboles. aspx?idCuadro=DOLAR_OBS_ADO. (Consulta 05 octubre 2019).

Baquedano, F., y W. Liefert. 2014. Market integration and price transmission in consumer markets of developing countries. Food Policy 44:103-114. doi: 10.1016/j. foodpol.2013.11.001.

Cottrell, A., y R. Lucchetti. 2007. Gretl: Gnu Regression, Econometrics and Time-series. URL http://gretl.sourceforge.net/. (Consulta 08 mayo 2017). 
Dempster, M., y K. Tang. 2016. Commodities. 1a․ ed. CRC Press, Boca Raton, Florida, USA.

Díaz, N., O. Melo, y F. Modrego. 2007. Dinámica de transmisión de precios y cambio estructural en el sector lácteo chileno. Agricultura Técnica 11:12-23.

Dutoit, L., K. Hernández, y C. Urrutia. 2010. Transmisión de precios en los mercados de maíz y arroz en América Latina. Serie Desarrollo Productivo $\mathrm{N}^{\circ}$ 190. CEPAL, Santiago, Chile.

Engle, R., y C. Granger. 1987. Co-integration and error correction: representation, estimation, and testing. Econometrica 2(55):251-276.

Engler, A., y L. Nahuelhual. 2003. Influencia del mercado internacional de lácteos sobre el precio nacional de la leche: un análisis de cointegración. Agricultura Técnica 53(4):41627. doi: $10.4067 / S 0365-28072003000400010$.

Engler, A., y L. Nahuelhual. 2008. Implications of the structural change in dairy products trade on milk price paid to producers in Chile. Chilean Journal of Agricultural Research 68:166-174. doi: 10.4067/S071858392008000200006.

Gaetano, F. 2015. Price transmission in the European tomatoes and cauliflowers sectors. Agribusiness 31(3):399-413. doi: 10.1002/ agr.21421.

Gujarati, D.N. 2004. Econometría. 4a. ed. Mc Graw Hill, México.

Hansen, B., y B. Seo. 2002. Testing for two-regime threshold cointegration in vector errorcorrection models. Journal of Econometrics 110(2002):293-318.

INE. 2019a. Estadísticas de Precio. IPC. Series empalmadas. Instituto Nacional de Estadísticas (INE). Disponible en http:// www.ine.cl/docs/default-source/precios/ ipc/series-empalmadas-y-historicas/serieshistoricas/ipc_general_indices_series_ historicas_empalme_1928_2009_base2008. xls?sfvrsn=bc9840d2_8. (Consulta 05 octubre 2019).

INE. 2019b. Estadísticas de Precio. IPC. Series empalmadas. Instituto Nacional de Estadísticas (INE). Disponible en http://www.ine.cl/docs/default-source/ precios/ipc/base-2009/series historicas/ ipc_general_referencial_diciembre2013. xlsx?sfvrsn=2da240d2_4. (Consulta 05 octubre 2019).
INE. 2019c. Estadísticas de Precio. IPC. Series empalmadas. Instituto Nacional de Estadísticas (INE). Disponible en http://www. ine.cl/docs/default-source/precios/ipc/base2013/series historicas/ipc_general_historico_ diciembre2018.xlsx?sfvrsn=2db640d2_58. (Consulta 05 octubre 2019).

Jaramillo, J., y E. Benítez. 2016. Transmisión de precios en el mercado mexicano e internacional de café (Coffea arabica L.): un análisis de cointegración. Agrociencia 50(7):931-944.

Labra-Hernández, J., J. Cabas-Monje, y J. Velasco-Fuenmayor. 2017. Efectos del precio internacional de la leche sobre el precio pagado a productor chileno: un análisis de cointegración. Revista Científica de la Facultad de Ciencias Veterinarias 27(6):385392.

Nahuelhual, L., y A. Engler. 2004. Efecto del precio internacional sobre el precio de la leche pagado a productor: transitorio o permanente?. Agricultura Técnica 64(4):388398. doi: 10.4067/S0365-28072004000400007.

Newton, J. 2016. Price transmission in global dairy markets. International Food and Agribusiness Management Review 19(B):113127.

Oficina de Estudios y Políticas Agrarias (ODEPA). 2017a. Precios. Series de tiempo. Precios al productor. Disponible en http://www.odepa. $\mathrm{cl} /$ precios/series-de-tiempo/. (Consulta 08 mayo 2017).

Oficina de Estudios y Políticas Agrarias (ODEPA). 2017b. Estadísticas. Comercio exterior. Matriz detallada de comercio exterior. Disponible en https://www.odepa.gob.cl/estadisticasdel-sector/bases-de-datos-comercio-exterior. (Consulta 05 octubre 2019).

Oficina de Estudios y Políticas Agrarias (ODEPA). 2017c. Publicaciones. Boletín. Boletín de la leche. Disponible en http://www.odepa.cl/ boletin/boletin-de-la-leche-5/ (Consulta 08 marzo 2017).

Popovic, R., B. Radovanov, y J. Dunn. 2017. Food scare crisis: the effect on Serbian dairy market. International Food and Agribusiness Management Review 20(1):113-127. doi: 10.22434/IFAMR2015.0051.

RStudio Team. 2016. RStudio: Integrated Development for R. RStudio, Inc., Boston, Massachusett, USA. Available at http://www. rstudio.com/. (Consulta 31 August 2019).

Serra, T.,yB.Goodwin.2003.Pricetransmissionand asymmetric adjustment in the Spanish dairy sector. Applied Economics 2003(35):18891899. doi: $10.1080 / 00036840310001628774$. 
Stephens, E., E. Mabaya, S. von Cramon-Taubadel, y C. Barrett. 2012. Spatial price adjustment with and without trade. Oxford Bulletin of Economics and Statistics 74(3):453-469. doi: 10.1111/j.1468-0084.2011.00651.x.

Valdés, R., S. von Cramon-Taubadel, J. Díaz, y A. Engler. 2011. The Chilean wheat market and its price support mechanism: a spatial market integration analysis. Revista de la Facultad de Ciencias Agrarias UNCUYO 43(2):47-58.

Wooldridge, J.M. 2006. Introducción a la econometría. Un enfoque moderno. 2a․ ed. Ed. Thomson, México. 